\title{
Hybrid Approach to Optimize the Cluster Flying Orbit for Fractionated Spacecraft Based on PSO-SQP Algorithm
}

\author{
Shenghui Wan ${ }^{1, a}$, Junling Song ${ }^{2, b}$, Jian Chen ${ }^{1, \mathrm{c}}$, Min $\mathrm{Hu}^{2, \mathrm{~b}}$ \\ ${ }^{1}$ China Satellite Maritime TTC Department, Jiangyin, China \\ ${ }^{2}$ Academy of Equipment, Beijing, China

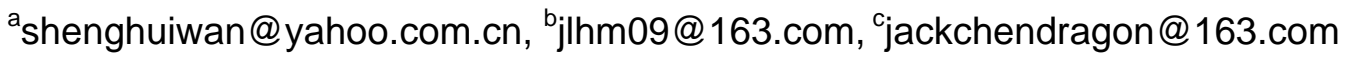

Keywords: Fractionated spacecraft; Cluster flying orbit; Relative orbital elements; Particle swarm optimization; Sequential quadratic programming.

\begin{abstract}
This template This paper investigates the optimal cluster flight orbit design issue for the fractionated spacecraft according to three main goals: keeping the cluster as stable as possible, preventing the collisions within the cluster, maintaining the inter-satellite distance within the maximum region. Firstly, the relative orbital elements are adopted to describe the relative motion. Then, the formation design requirements are formulated in terms of the relative orbital elements, the constrained optimization problem is solved using the hybrid particle swarm optimization algorithm integrated with sequential quadratic programming local search. The simulation results show that the hybrid PSO-SQP algorithm is effective.
\end{abstract}

\section{Introduction}

Fractionated spacecraft has been well concerned in recent five years because of its unique technical merits. The concept of the fractionated spacecraft is to separate the traditional monolithic satellite into free-flying service modules and different payloads, which are connected by self-organizing network wirelessly. DARPA began a program called the System F6 (Future, Fast, Flexible, Fractionated, Free-Flying Spacecraft) in 2008. The fractionated spacecraft usually consist of tens and scores of modules and the modules often operate in close proximity, which make the cluster flying orbit design issue more challenging ${ }^{[1]}$.

The requirements of fractionated spacecraft formation design can be summarized as follows: the propellant should be minimized, the relative motion should be stable to avoid collisions, the inter-satellite distance should be maintained to ensure the inter-satellite communication and wireless power transfer. Relative orbital elements were demonstrated during the GRACE, PRISMA, and TanDEM-X missions. This paper adopts the relative orbital elements to formulate the goal function and constraint functions. Generally, the particle swarm optimization (PSO) algorithm has a global search at the beginning whereas a local searching ability near the end of the run. Therefore, the sequential quadratic programming (SQP) technique is used to reduce computation time and to improve convergence performance.

The organization of this paper is as follows: firstly, the relative orbital elements are defined and the cluster configuration description parameters are proposed; secondly, we formulate the optimal cluster flying orbit design issue based on the cluster configuration description parameters, the objective function and the nonlinear constraints are put forward; then, the optimal problem is solved in the framework of PSO-SQP algorithm, we utilize the PSO algorithm to find the potential initial guess point as the input for the SQP algorithm; finally, the numerical simulations of 7 satellites are carried out to validate the proposed hybrid optimization approach.

\section{Description of the general formation configuration}

The Keplerian orbital elements are $a, e, i, \Omega, \omega$, and $u$, which correspond to the semi-major axis, eccentricity, inclination, right ascension of the ascending node, argument of perigee, and mean 
argument of latitude ( $u=\omega+M$, where $M$ is the mean anomaly), respectively. Spacecraft- 1 is the master satellite, and Spacecraft-2 is the deputy satellite. For near-circular satellite orbits, the relative eccentricity vector can be defined as follows:

$$
\begin{aligned}
& \Delta \boldsymbol{e}=\left[\begin{array}{l}
\Delta e_{x} \\
\Delta e_{y}
\end{array}\right]=\delta e\left[\begin{array}{c}
\cos \theta \\
\sin \theta
\end{array}\right]=e_{2}\left[\begin{array}{l}
\cos \omega_{2} \\
\sin \omega_{2}
\end{array}\right]-e_{1}\left[\begin{array}{c}
\cos \omega_{1} \\
\sin \omega_{1}
\end{array}\right] \\
& \theta=\arctan \left(\Delta e_{y}, \Delta e_{x}\right)
\end{aligned}
$$

where $\delta e$ represents the amplitude of $\Delta \boldsymbol{e}$ and $\theta$ defines the initial phase angle of the in-plane motion.

The inclination vector $\Delta \boldsymbol{i}$ can be defined using the law of sines and cosines for the spherical triangle ${ }^{[2]}$ :

$$
\begin{aligned}
& \Delta \boldsymbol{i}=\left[\begin{array}{l}
\Delta i_{x} \\
\Delta i_{y}
\end{array}\right]=\delta i\left[\begin{array}{l}
\cos \varphi \\
\sin \varphi
\end{array}\right] \approx\left[\begin{array}{c}
\Delta i \\
\Delta \Omega \sin i_{1}
\end{array}\right] \\
& \varphi=\arctan \left(\Delta i_{y}, \Delta i_{x}\right)
\end{aligned}
$$

where $\Delta i=i_{2}-i_{1}, \Delta \Omega=\Omega_{2}-\Omega_{1}, \delta i$ represents the amplitude of $\Delta \boldsymbol{i}$, and $\varphi$ defines the initial phase angle of the cross-track plane motion.

For a near-circular reference orbit, the relative motion of the formation flying satellites can be described by the following equations:

$$
\left\{\begin{array}{l}
x=\Delta a-p \cos (u-\theta) \\
y=2 p \sin (u-\theta)+l \\
z=s \sin (u-\varphi)
\end{array}\right.
$$

where $\{p, s, \alpha, \theta, l\}$ are the five general formation configuration description parameters; $p=a \delta e$ represents the semi-minor axis of the relative in-plane ellipse; $s=a \delta i$ denotes the cross-track amplitude; $\alpha=\theta-\varphi$ defines the relative initial phase angle between the in-plane and cross-track plane motions; and $\theta$ is the initial phase angle of the in-plane motion. $\Delta u=u_{2}-u_{1}, l=a(\Delta u+\Delta \Omega \cos i)-\frac{3}{2}\left(u-u_{0}\right) \Delta a, u_{0}$ is the initial mean argument of latitude of the deputy satellite, and $l$ represents the along-track offset of the center of the in-plane motion. An example trajectory is shown in Fig. 1.
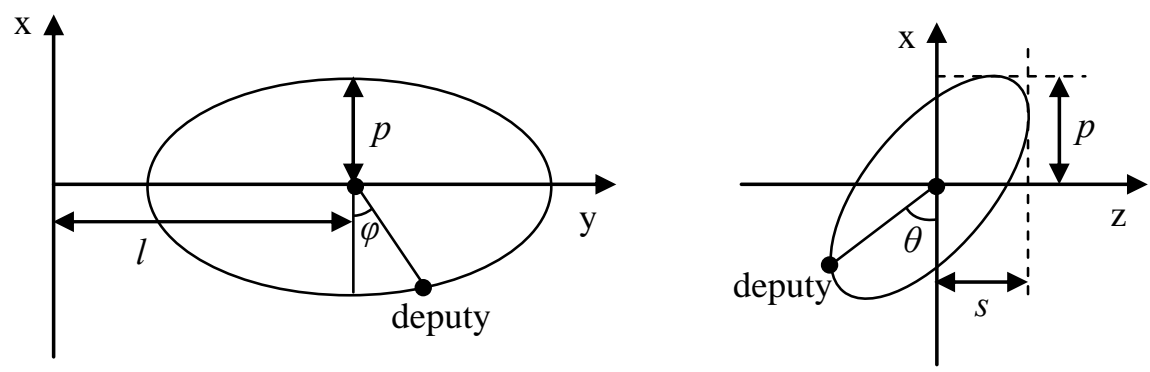

Fig. 1. Example of a relative motion in a near-circular reference orbit.

\section{Optimization Problem}

\section{Design Variables}

The goal of the cluster flying orbit design is to design the formation configuration description parameters $\{p, s, \alpha, \theta, l\}$ for all the member satellites. The along-track offset $l$ can be assumed to equal zero for the nominal configuration. In order to eliminate the secular drift caused by the difference in $J_{2}$ perturbation, we can adopt the $J_{2}$ invariant relative orbit. However, the $J_{2}$ invariant relative orbit requirements are too strict, which may result in impractically large relative orbits. Therefore, we adopt the partly $J_{2}$ invariant relative orbit to reduce the main influences of the $J_{2}$ perturbations. We 
force the inclinations of the fractionated spacecraft to be equal, which greatly reduces the influences the $J_{2}$ perturbation. In other words, $\varphi_{i}=\pi / 2$ or $\varphi_{i}=3 \pi / 2, i=1,2 \cdots N$.

Therefore, the design variables are $\left\{p_{i}, s_{i}, \theta_{i}\right\}$, where $i$ represents the number of the member satellites.

\section{Objective Function.}

The combination of the relative distances in the $x o z$ plane and the three-dimensional relative distances are chosen as the optimization performance index ${ }^{[3]}$.

$$
J=\sum_{\substack{j=1 \\ j \neq i}}^{N} \sum_{i=1}^{N}\left[C_{1}\left(\left(r_{x o z}^{\min }\right)_{i j}-d_{x o z}^{s a f e}\right)^{2}+C_{2}\left(\left(r_{x y z}^{\max }\right)_{i j}-d_{x y z}^{\max }\right)^{2}\right]
$$

where $C_{1}$ and $C_{2}$ are the weighting coefficient, $r_{x o z}^{\min }$ is the relative distance in the xoz plane, $d_{x o z}^{\text {safe }}$ is the minimum safe distance predefined to guarantee the passive relative orbit, $r_{x y z}^{\max }$ is the relative distance between any two satellites, $d_{x y z}^{\max }$ is a predefined maximum distance which guarantee the member satellites can communicate with each other regularly. $\mathrm{N}$ is the number of the member satellites.

\section{Nonlinear Constraints}

Collision Avoidance Requirement:

Substituting Eq.(5) into Equation $r_{X O z_{-} \min }=\sqrt{x^{2}+z^{2}}$, we derive the minimum distance in the cross-track plane based on the proposed configuration parameters, the safety of the cluster flying satellites is guaranteed even in the presence of the along-track uncertainty ${ }^{[4]}$.

$$
r_{X O Z_{-} \min }=\sqrt{\frac{p^{2}+s^{2}-\sqrt{p^{4}+s^{4}-2 p^{2} s^{2} \cos 2 \alpha}}{2}}
$$

Maximum Inter-Satellite Distance Requirement:

Substituting Eq.(5) into Equation $r_{X Y Z_{\text {max }}}=\sqrt{x^{2}+y^{2}+z^{2}}$, we derive the maximum relative distance between the any two member satellites based on the proposed configuration parameters, which guarantee all member satellites are in the communication range.

$$
r_{X Y Z_{-} \max }=\sqrt{\frac{5 p^{2}+s^{2}+\sqrt{9 p^{4}+s^{4}+6 p^{2} s^{2} \cos 2 \alpha}}{2}}
$$

\section{Hybrid PSO-SQP Algorithm}

The PSO algorithm is one of the evolutionary computation techniques introduced by Kennedy and Eberhart in 1995. The basic PSO algorithm is ${ }^{[5]}$ :

$$
\begin{aligned}
& v_{i d}^{k+1}=\omega \cdot v_{i d}^{k}+c_{1} \cdot \operatorname{rand}() \cdot\left(P_{i d}-x_{i d}^{k}\right)+c_{2} \cdot \operatorname{rand}() \cdot\left(P_{g d}-x_{i d}^{k}\right) \\
& x_{i d}^{k+1}=x_{i d}^{k}+v_{i d}^{k+1}
\end{aligned}
$$

The SQP is a nonlinear programming method, which is very fit for the nonlinear constraint programming issue ${ }^{[6]}$ :

$$
\begin{aligned}
& \min _{d \in R^{n}} \frac{1}{2} d^{T} H_{k} d+\nabla f\left(x_{k}\right)^{T} d \\
& \text { s.t. } \nabla g\left(x_{k}\right)^{T} d+g\left(x_{k}\right) \leq 0
\end{aligned}
$$

We propose a hybrid optimization approach, which utilize the PSO to find the initial guess value for the SQP, the SQP technique is used to reduce computation time and to improve convergence performance. We use a linear decay rule to adapt the inertia weight, and use SQP technique when the convergent value satisfy the predefined threshold. The operational flow of the PSO-SQP algorithm is shown as Fig. 2. 


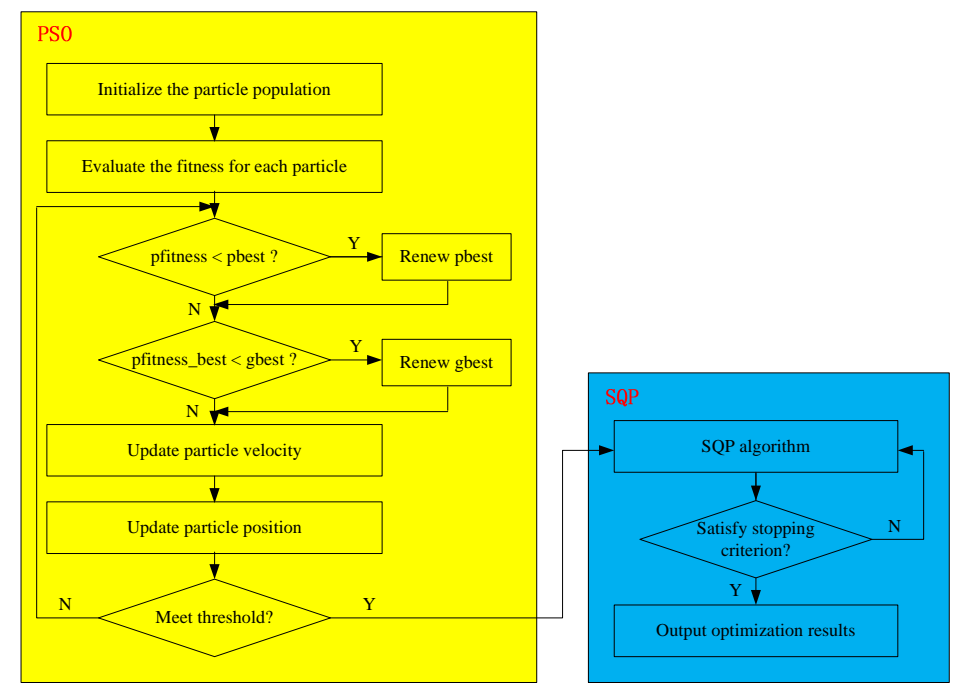

Fig. 2. Operational flow of the PSO-SQP algorithm.

\section{Numerical Simulations and Analysis}

Seven modules are simulated, the minimum distance in the cross-track plane is $100 \mathrm{~m}$, the maximum communication distance is $2000 \mathrm{~m}$. The particle number is 40 , the inertial weight vary from 0.9 to 0.4 , the learn factors are 2, 2, respectively. The threshold for the switching of PSO to SQP is 0.01 . The orbital elements of the reference satellite is $a=6878 \mathrm{~km}, e=0.0001, i=98.4^{\circ}, \Omega=0.2497^{\circ}$, $\omega=30^{\circ}$, and $M=60^{\circ}$.

The optimal configuration design results of the 7 satellites are shown in Table 1.

Table 1 Optimal configuration design results of the 7 satellites.

\begin{tabular}{cccccccc}
\hline & Sat1 & Sat2 & Sat3 & Sat4 & Sat5 & Sat6 & Sat7 \\
\hline$p / \mathrm{m}$ & 0 & 713.979 & 295.087 & 458.176 & 420.170 & 913.632 & 728.127 \\
$s / \mathrm{m}$ & 0 & 728.510 & 1652.488 & 1777.860 & 270.765 & 602.858 & 1371.688 \\
$\theta /^{\mathrm{o}}$ & 0 & 11.255 & 60.233 & 88.255 & 58.177 & 39.291 & 96.833 \\
\hline
\end{tabular}

Figure 3 shows the three-dimensional formation configuration, Fig. 4 shows the projected formation configuration in the $x o z$ plane, Fig. 5 shows the relative distances among the 7 satellites in the $x o z$ plane, while Fig. 6 shows the three-dimensional relative distances among all satellites.

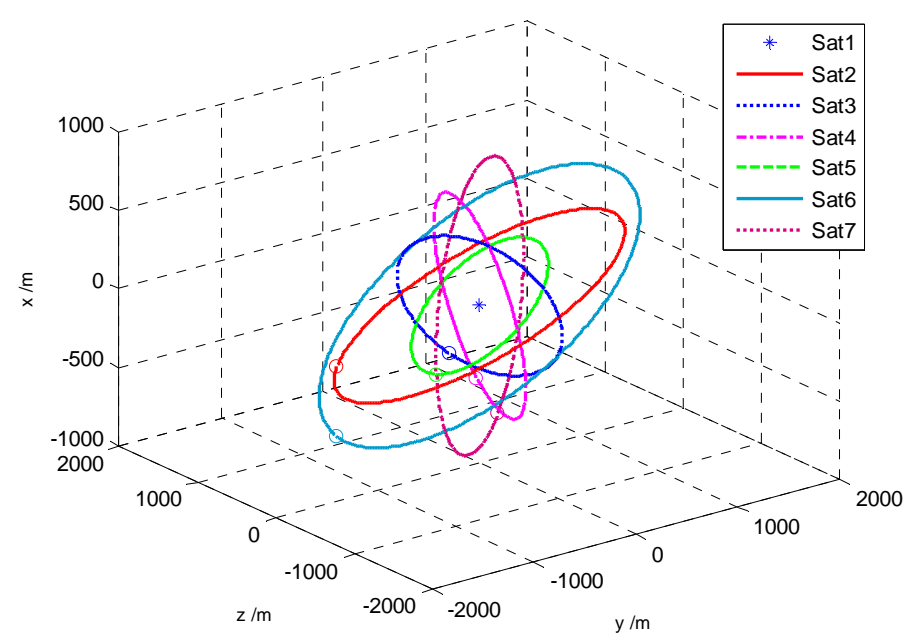

Fig. 3. The three-dimensional formation configuration. 


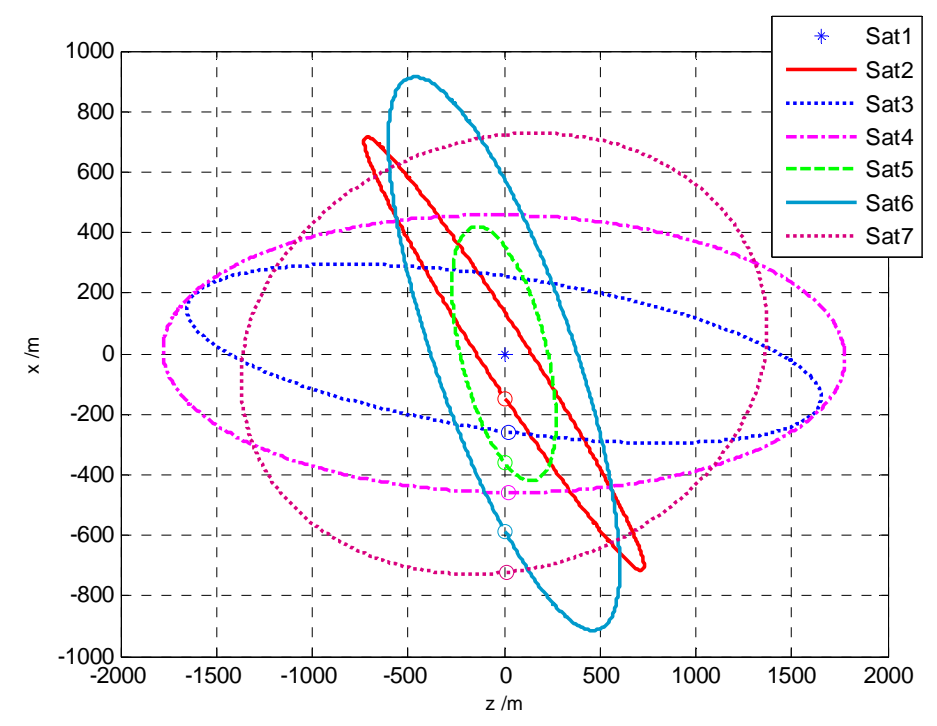

Fig. 4. The projected formation configuration in the $x o z$ plane.

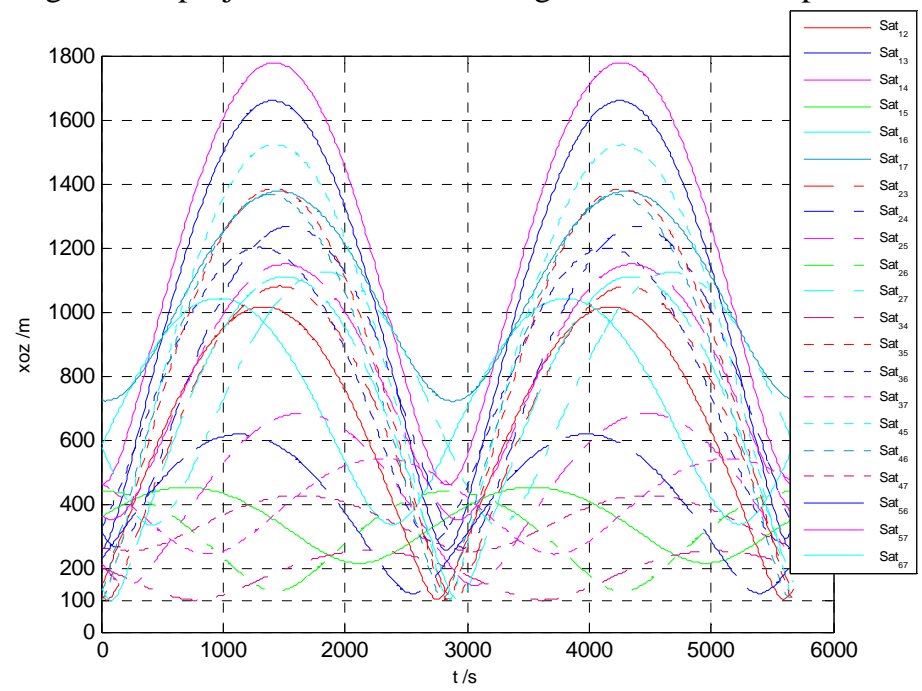

Fig. 5. The relative distances in the xoz plane.

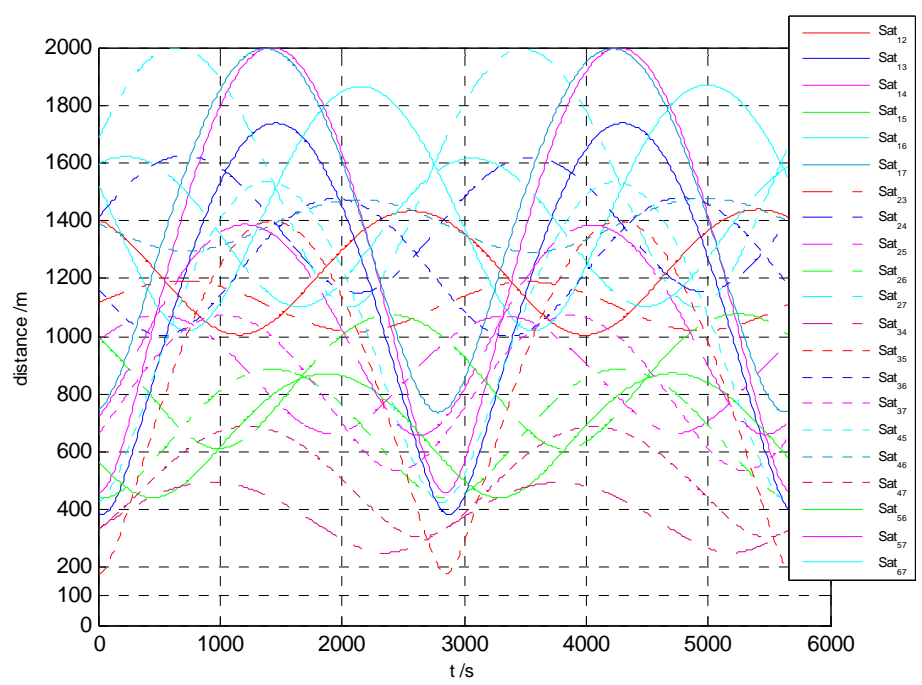

Fig. 6. The three-dimensional relative distances.

As shown in Fig. 5, the minimum distance among all satellites in the $x o z$ plane is always larger than 100m. As shown in Fig. 6, the maximum distances among all satellites satisfy the maximum communication constraint $2000 \mathrm{~m}$. 


\section{Summary}

The current paper presents a hybrid optimization approach for the cluster flying orbit design of the fractionated spacecraft. The contributions of this paper can be summarized in two folds: 1) We establish the mathematical models of the problem, including the design variables, objective function and the nonlinear constraints, the derived formula of the constraints are based on the presented formation description parameters. 2) We adopt the PSO technique to find the initial guess value for the SQP, the SQP technique is used to reduce computation time and to improve convergence performance. The conclusion is that the PSO-SQP algorithm is valid and efficient for the optimal cluster flight orbit design issue of the fractionated spacecraft.

\section{References}

[1] G.Q. Zeng, M. Hu, H. Yao. Relative Orbit Estimation and Formation Keeping Control of Satellite Formations in Low Earth Orbits [J]. Acta Astronautica. 2012,76: 164-175.

[2] J.S. Ardaens,S. D'Amico. Spaceborne Autonomous Relative Control System for Dual Satellite Formations [J]. Journal of Guidance, Control, and Dynamics, 2009, 32(6):1859-1870.

[3] J.H. Wang, S. Nakasuka. Cluster Flight Orbit Design Method for Fractionated Spacecraft [J]. Aircraft Engineering and Aerospace Technology, 2012, Vol. 84 Iss: 5.

[4] G.Q. Zeng, M. Hu. Collision Monitoring and Optimal Collision Avoidance Maneuver for Formation Flying Satellites [J]. Aircraft Engineering and Aerospace Technology. 2012,84(6):413-422.

[5] J. Kennedy, R. Eberhart. Particle Swarm Optimization [C]. Proceedings of the IEEE International Conference on Neural Network, Perth, Australia, 1995: 1942-1948.

[6] R.B. Wilson. A simplicial Algorithm for Concave Programming[D]. Boston: Graduate School of Business Administration, Harvard University, 1963. 